\title{
Commentary
}

\section{DORSCON Orange: An Approach to Challenges in a Busy Antenatal Diagnostic Centre in the Midst of a Global Pandemic}

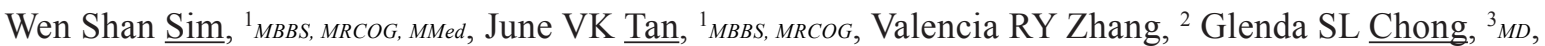
Chee Wai $\underline{\mathrm{Ku}},{ }^{3}$ MD, Kok Hian $\underline{\mathrm{Tan}},{ }^{1}$ MBBS, FRCOG, FAMS, Hak Koon Tan, ${ }^{3}$ MBBS, FRCOG, FAMS, George SH $\underline{\text { Yeo }},{ }^{1}$ MBBS, FRCOG, FAMS, Shephali Tagore, ${ }^{1}$ MBBS, MD, FRCOG

\begin{abstract}
The WHO declared the coronavirus disease 2019 (COVID-19) a global pandemic on 11 March 2020. Lessons from SARS epidemic led Singapore to develop stringent infection control protocols in preparation for future pandemics. However, unlike SARS, COVID-19 appears to be more transmissible and is predicted to continue for longer. As of 14 June 2020, there have been 40,197 positive cases with 26 deaths in Singapore, and KK Women's and Children's Hospital (KKH) has managed a total of 73 cases. Obstetrics ultrasound is an indispensable medical service and must continue to operate during a pandemic. A key balance must be struck between keeping patients and healthcare workers safe while being able to provide quality and prompt obstetric care. Our Antenatal Diagnostic Centre (ADC) in KKH developed new strategies to adapt to the pandemic when the national Disease Outbreak Response System Condition (DORSCON) was raised from yellow to orange on 7 February 2020 . In this paper, we discuss our clinical workflow to reduce the risk of transmission amongst patients and staff while minimising disruption to our services.
\end{abstract}

Ann Acad Med Singap. 2020;49:677-83

\section{Maternal and Fetal Department: Antenatal Diagnostic Centre (ADC)}

KK Women's and Children's Hospital (KKH) is the largest tertiary Women's and Children's hospital in Singapore with approximately 11 to 12,000 deliveries per year.

The Antenatal Diagnostic Centre (ADC) provides well-coordinated, specialised multidisciplinary Fetal Medicine Clinic (FMC) where maternal-fetal medicine (MFM) specialists, obstetricians, genetic counselors, neonatologists and ultrasonographers work together to provide each patient with a high level of quality care in the management of women with complicated pregnancies and fetal anomalies. We perform an average of 223 scans per day, amounting to an average of 4600 scans per month in 2019.

ADC comprises of 16 scan rooms, 2 of which are used by lead sonographers for fetal anomalies and complex pregnancies. One room is dedicated to diagnostic procedures such as Amniocentesis and Chorionic Villous Sampling. A Fetoscopy suite is reserved for advanced interventions such as Fetoscopic

\footnotetext{
${ }^{1}$ Antenatal Diagnostic Centre, Department of Maternal and Fetal Medicine, KK Women's and Children's Hospital, Singapore

${ }^{2}$ Yong Loo Lin School of Medicine, National University of Singapore, Singapore

${ }^{3}$ Division of Obstetrics and Gynecology, KK Women's and Children's Hospital, Singapore

Address for Correspondence: Dr Sim Wen Shan, Yong Loo Lin School of Medicine, 1E Kent Ridge Road, Singapore 119228

Email: sim.wen.shan@kkh.com.sg
} 
Laser Photocoagulation, chest shunts and cord coagulation etc. ADC is situated on the second floor next to delivery suite.

A sister unit, the Antenatal Monitoring Center (AMC), is located on a separate floor nearer the outpatient clinics and comprises 3 scan rooms. The main role of the AMC is to provide routine obstetrics scans.

\section{Challenges in ADC And Strategies to Overcome Them}

\section{Manpower Reallocation in Teams}

The manpower strategy of ADC was discussed over many leadership meetings and it was decided that team segregation approach would be best (Table 1). The aim was to preserve an active manpower unit in the event of an unprotected exposure to a COVID-19 patient. ADC staff including doctors, sonographers and patient care assistants were divided into two teams which would operate in two physically separate areas of ADC. There is no physical interaction between these 2 teams at any point in time. However, the 2 teams communicate closely through teleconferencing, electronic messaging and secure electronic chat groups. Each segregated team consists of 1 principal sonographer, 4 sonographers with a variety of skill sets, 2 MFM specialists and 1 resident. The Antenatal Monitoring Center (AMC) provides a contingent venue in case ADC is affected by COVID-19 outbreak. Manpower shortage during a pandemic potentially leads to burnout and fatigue of remaining medical staff, which will further aggravate manpower issues. ${ }^{1,2}$

\section{Highly Transmissible Nature of COVID-19 and Screening Tools}

In view of our large number of pregnant women, there is a high risk of transmission if robust measures are not in place. Social distancing was enforced in queues and sitting areas of ADC, ensuring patients are at least 1-2 meters from each other. Regular meetings traditionally attended by multidisciplinary teams to discuss complex cases are limited to a smaller group to reduce transmission. All ADC staff are required to wear surgical facemasks, and underwent training for optimal use of personal protective equipment (PPE) arranged by the infectious diseases team. Quarantine procedures and monitoring for symptoms was also reiterated. It is inevitable that performing an ultrasound poses a unique challenge for social distancing. A sonographer may be in close contact with a patient for up to 30 to 45 minutes for fetal anomaly scans. To mitigate this risk, all sonographers are required to wear surgical facemasks and to practice strict hand hygiene and personal hygiene during all the ultrasound examinations. Partners are not allowed into the scan rooms during this period.

The need for stringent screening procedures was reinforced during the SARS era. ${ }^{3}$ Triage counters were set up at all entry points to the hospital. Patients and visitors were required to check in to the hospital with "SafeEntry", the national digital system for contact tracing. Their temperatures were measured at these triage counters. A unique coloured sticker was provided to signify their designated area of visitation. A concise declaration form was designed and continuously updated in alignment with the latest national travel restrictions and quarantine orders (Appendix 1). Additional manpower was deployed to aid this process. Guidance for accompanying visitor was created (Figure 1). Electronic billing minimised physical contact and prevented overcrowding in the counter areas.

\section{Allowing Obstetric Scans and Procedures to Continue}

A total of 30,659 scans (with a mean of 2,555 scans per month) were performed in the ADC in 2019, and a further 7,501 scans (with a mean of 2,500 scans per month) were performed in 2020 (Figure 2). The average number of scans per sonographer per month was 213 in 2019 and 208 in 2020 (Supplementary Table 1). A balance must be struck between the provision of critical ultrasound scans and reducing the risk of exposure between healthcare workers and patients. Some elective ultrasounds were postponed or cancelled if the risk of exposure and infection seem to outweigh the benefit of investigations. ${ }^{4}$ Women who were screened positive for any item on the declaration form would have their scan appointments reviewed for rescheduling. Our recommendation for scan rescheduling is attached (Figure 3). If the appointment cannot be rescheduled due to clinical reasons, the scan will proceed in the isolation room. To date, 130 scans have been performed in our isolation facility. Since DORSCON orange, 13 First Trimester Screening (FTS) have been cancelled and replaced by Non-invasive Prenatal Test (NIPT) which can be performed outside the FTS gestational week range, and 6 were relisted.

Adjustments were made to our service provision during DORSCON orange. Patients with a fetus with thickened nuchal translucency (NT) of $>97 \%$ on FTS were traditionally given an appointment for cardiac scan at 16 weeks' gestation, but this service was now limited to cases with NT $\geq 3.5 \mathrm{~mm}$. A detailed screening scan remained routine for all FTS cases with NT $>97 \%$. Patients requiring cervical length monitoring was offered screening from 16 weeks onwards instead of 14 weeks (Figure 3). ${ }^{5}$ After introduction of the above guidelines, 
Table 1. The Challenges in ADC and Strategies to Overcome Them

\begin{tabular}{ll}
\hline & Challenges \\
\hline 1. & $\begin{array}{l}\text { Manpower reallocation } \\
\text { in teams }\end{array}$ \\
& \\
\hline 2. & Highly transmissible \\
& nature of COVID -19 \\
& \\
& Screening of large \\
volume of patients and \\
visitors prior to entering \\
ADC
\end{tabular}

\section{Strategies}

a. ADC area is divided into 2 separate areas.

b. MF team is also divided into 2 teams and is segregated. Each team consists of sonographers and Maternal Fetal Medicine specialists and residents. Each sonographer team consists of a lead sonographer capable of doing complex scans such as fetal anomaly, on top of simple scans and first trimester screening.

c. Dedicated area is reserved for fetal interventions such as amniocentesis, chorionic villus sampling etc.

d. Fetoscopy suite is reserved for advance fetal interventions such as laser, photocoagulation etc.

e. Downsizing of meetings attended by multidisciplinary staff to discuss complex fetal medicine cases.

f. Doctors in ADC are contributing by multi-tasking and helping with sonographic work in ADC on top of their clinical and surgical work.

a. Front line staff don surgical masks during triage with strict compliance to hand hygiene.

b. All staff in ADC who have patient contact to abide by frequent hand washing and 5 moments of hand hygiene.

c. Maintain 1-2-metre social distancing in waiting areas and. Electronic billing to avoid queues.

a. Triage counters at all entry points of the hospital. Compulsory temperature screening by infrared sensor.

b. Screening of all patients and accompanying visitors for presence of respiratory symptoms or fever based on travel history, occupation and significant contact history.*

c. Screen positive patients will have non-urgent appointments reviewed for rescheduling.

d. If accompanying person is a healthcare worker involved in the care of COVID-19 patients, he/she is discouraged from entering the scan room. Otherwise donning an N95 mask is necessary.

Note: Travel restrictions and Stay-Home-Notices are imposed by the Singapore Government. Any false declaration of travel history or breach of Stay-Home-Notice is reportable to Ministry of Health (MOH).

*Patients undergoing ultrasound scan from $27^{\text {th }}$ March 2020 are discouraged from bringing accompanying visitors into the scan room, in line with government policy of "social distancing".

\section{Allowing obstetric scans and procedures to continue}

a. Pregnant patients with known risk factors and those with mild or asymptomatic COVID-19 infection will have antenatal visits and routine ultrasound assessments (excluding time-sensitive scans) delayed by 14 days. Teleconsult is preferred.

b. For patients with positive screening history, FTS scans will be cancelled if NIPT has been done and screening scan is rescheduled to a maximum of 23 weeks' gestation. Individual cases are discussed and documented in medical records.

c. For patients with positive screening history, scans are done in the isolation room with a dedicated sonographer in-charge using PPE equipment.

d. For scans done in isolation room, accompanying persons are strictly prohibited.

e. Abdominal transducers are cleaned after each examination with alcohol-based wipes. Transvaginal transducers are covered with 2 condoms, and after every use, sterilised with ANTIGERMIX®.

\section{Adjustments in service} provision

a. Fetal cardiac scans are limited to patients with a fetus with thickened nuchal translucency (NT) $\geq 3.5 \mathrm{~mm}$ ( $>99 \%$ percentile) instead of an NT of $>97 \%$ percentile.

b. A detailed screening scan remains routine for all FTS cases with NT $>97 \%$.

b. Patients requiring cervical length monitoring will be offered screening from 16 weeks onwards instead of 14 weeks

6. Maintaining an isolation facility

a. All attending medical staff don PPE (including a surgical cap, eye protection with goggles, N95 mask, gloves, isolation gown and shoe cover)

b. Isolation rooms are sterilised after each use. Hard surfaces are cleansed by chlorine tablets dissolved in solution. For scan machines and transabdominal probes, WIP'ANIOS excel wipes are used. Transvaginal probes are disinfected with ANTIGERMIX ${ }^{\circledR}$, a UV-C technology which performs high level disinfectant in 3 minutes. The housekeeping dressed in PPE will do another round of general cleaning. The room is then left for 1 hour before next use.

c. Medical staff caring for suspected, probable, and confirmed cases of COVID-19 should be monitored closely for respiratory symptoms or fever.

d. Medical staff who have been exposed unexpectedly without PPE should be quarantined or self-isolate for 14 days.

7. Other challenges on the

a. Recognise the need for and provide emotional and psychological support for staff to prevent burn-out. ground in the midst of an evolving pandemic

ADC: Antenatal diagnosis centre; FTS: First trimester screening; MF: Maternal-fetal; NIPT: Non-invasive prenatal test; NT: Nuchal translucency 


\begin{tabular}{|c|r|}
\hline Mitigating transmission risks & Manpower reallocation in teams \\
\hline $\begin{array}{l}\text { 1. All medical staff to wear surgical masks } \\
\text { and strictly comply to } 5 \text { steps of hand }\end{array}$ & $\begin{array}{l}\text { 1. } \begin{array}{l}\text { Segregation of manpower into } 2 \text { teams } \\
\text { consisting of sonographers, Maternal }\end{array} \\
\begin{array}{l}\text { hygiene } \\
\text { 2. Social distancing enforced for patients in } \\
\text { queues and waiting areas }\end{array}\end{array}$ \\
\hline
\end{tabular}

Screening for COVID-19 (all patients and accompanying persons)

1. Travel to any countries out of Singapore within 14 days OR

2. History of contact with any hospital abroad OR history of close contact with a confirmed case of COVID-19 within 14 days before onset of illness

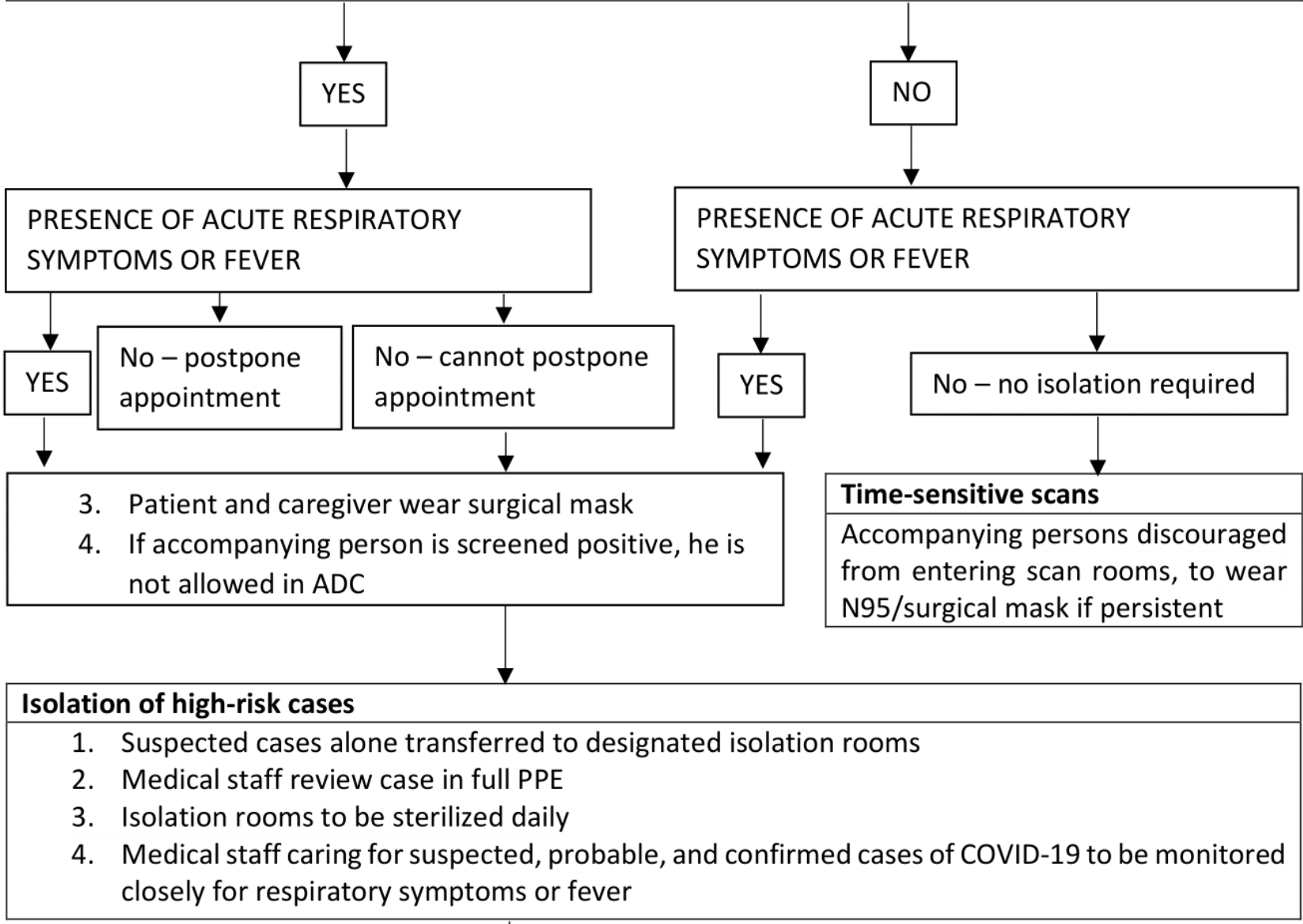

\section{Obstetric ultrasound scans and procedures}

1. Dedicated sonographer in-charge of isolation room carries out scans and procedures in full PPE

2. Surfaces of transducers are cleaned and disinfected according to manufacturer specifications, taking note of the recommended 'wet time' for wiping transducers and other surfaces with disinfection agents.

3. Protective covers are be used for probes and cables in cases where there are infected skin lesions or when transvaginal scan is necessary 


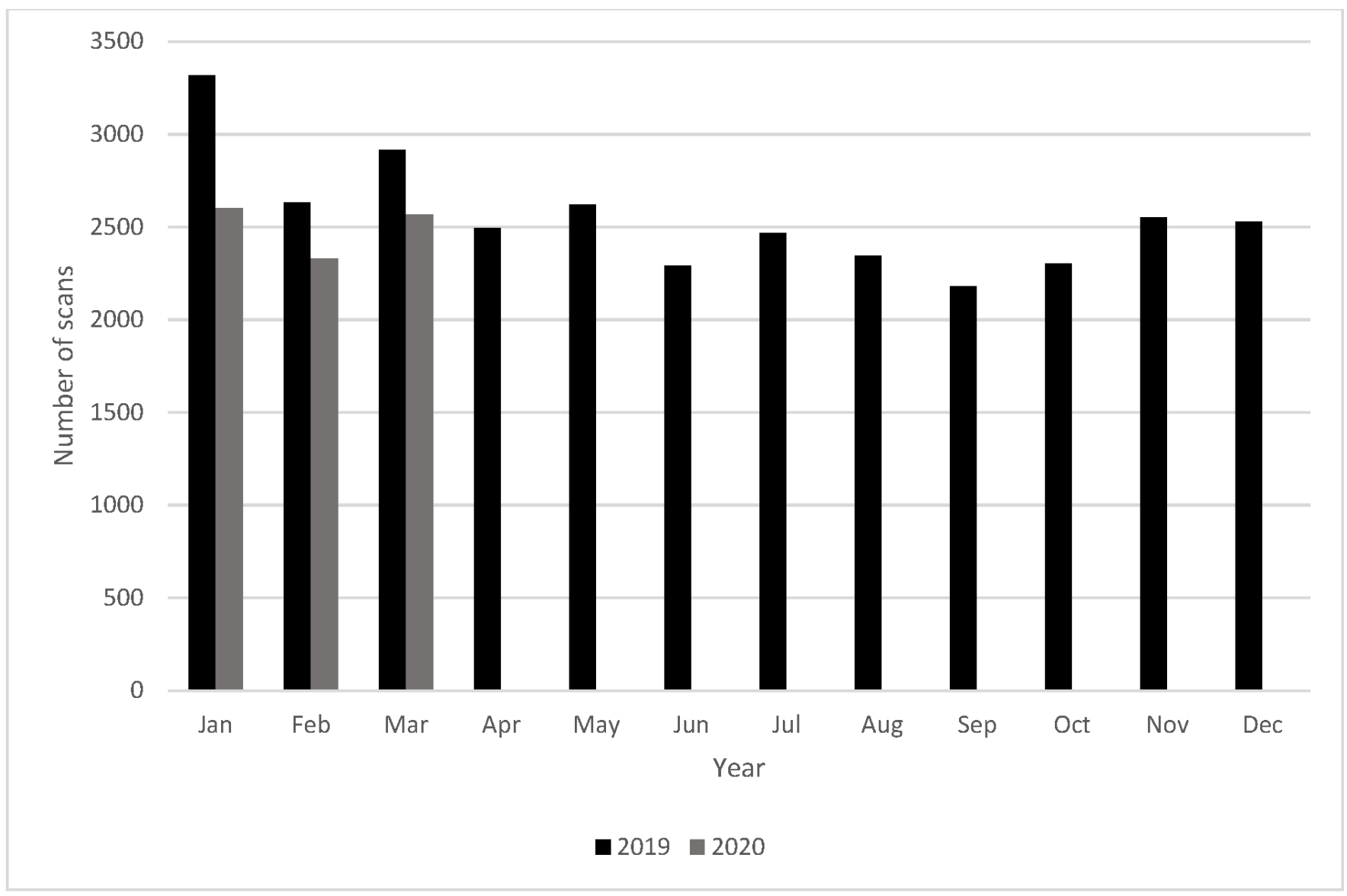

Fig. 2. Overview of Number of Scans Done in ADC in 2019 and 2020

our 16th week cardiac scans decreased from an average of 40 cases per month to 1 case in February and 0 cases in March.

As a tertiary center, second opinion scans and external referrals were still accepted. However, these patients and visitors were subjected to our triaging and visitation policies. There were no overseas referrals since travel restrictions were imposed.

Existing cleaning and disinfection protocols for the ultrasound transducers were strictly reinforced (Table 1$){ }^{6}$

\section{Maintaining an Isolation Facility}

Swift isolation of suspect cases decreases the likelihood of further spread as seen in the outbreak of SARS in $2003 .^{8}$ A dedicated room furthest away from the main area of ADC was chosen for isolation. The room is minimally furnished and has a separate route leading to it, avoiding the main area of ADC. The logistics as to how the patient would reach the isolation room was thoroughly planned. A simulation exercise was held. ADC staff who managed isolation cases were required to wear full PPE. Standard procedures for disinfection were followed. ${ }^{7}$ After each patient was scanned in the isolation room, the room was thoroughly disinfected (Table 1).

\section{Preventing Burn-Out Among ADC Staff (Self-Care Pro- gramme)}

Scan cancellations and denial of visitors had led to stressful confrontations between patients and staff in ADC. The hospital instituted a self-care programme which encouraged staff to pause and be refreshed through mindfulness and care for self. Healthcare workers were also given hotlines to call should they require any emotional support.

It is also of note that the absenteeism rate of sonographers had increased in the month of March after DORSCON Orange was declared. The percentage of number of working days absent per month amongst 15 sonographers went up to $58.3 \%$ in March 2020 from $17.8 \%$ in February 2020 . A potential cofounding factor may be due to a national policy of mandatory 5-day medical certificate (MC) leave for any acute respiratory symptoms. However, the element of burnout cannot be excluded. Burnout and absenteeism form a vicious cycle. The etiology of burnout can be 
KK Women's and

Children's Hospital

DORSCON ORANGE:

GUIDELINE FOR ANTENATAL DIAGNOSTIC CENTRE (ADC)/ ANTENATAL MONITORING CENTRE (AMC)

\section{Outpatient Service Provision}

1. All women who are screened positive for any of the following on the KK Women's and Children's Declaration form (ANNEX 1) will be reviewed for rescheduling of appointment. If appointment cannot be rescheduled due to clinical reasons, the ultrasound scan will proceed in the isolation room.

2. Recommendation for scan rescheduling

\begin{tabular}{|l|l|}
\hline Scan & Recommendation \\
\hline First trimester Screening (FTS) & $\begin{array}{l}\text { RTS scan will be cancelled if NIPT is done. } \\
\text { (Unless previous structural anomaly or any other concerns) } \\
\text { NIPT or Maternal Serum Screening will be offered if FTS scan } \\
\text { Cannot be rescheduled before } 14^{+0} \text { weeks. }\end{array}$ \\
\hline $\begin{array}{l}\text { Ultrasound Screening } \\
\text { (Fetal Anomaly) }\end{array}$ & $\begin{array}{c}\text { Rescheduled to a maximum of } 23 \text { weeks gestation. } \\
\text { Special considerations: } \\
\text { 1. High risk patients with co-morbidities such as } \\
\text { preeclampsia, diabetes or complicated medical } \\
\text { disorders } \\
\text { 2. Any concerns about growth or measurements on } \\
\text { bones i.e. achondroplasia. }\end{array}$ \\
\hline $\begin{array}{c}\text { Please discuss the urgency of US growth with an MF } \\
\text { consultant. }\end{array}$
\end{tabular}

Fig. 3. Recommendations of ADC Protocol During DORSCON Code Orange 
multifactorial. This may result from increased workload, stress of meeting expectations of patients while working with constraints, having to don uncomfortable PPE while scanning, extra sterilisation procedures and the worry of contracting COVID-19 from close contact during scans. Further studies can be done to look for the presence of burnout amongst ADC staff, its contributary factors and the effect of burnout on the absenteeism rate in ADC.

\section{Conclusion}

Obstetric ultrasound is an essential hospital service and must continue operations during a pandemic. Akey balance must be struck between keeping patients and healthcare workers safe, and providing quality and prompt obstetric care. The existing strategies may need to be reviewed continually while we monitor the evolving pandemic situation. We hope to remain flexible and to be able to adapt quickly to evolving situations, while ensuring our staff remain resilient.

\section{Acknowledgements}

The authors would like to thank Ms Padma Lata, Ms Dina Tan and Ms Cindy Liew of the KKH Maternal Fetal Medicine department for their assistance in data provision. We would also like to acknowledge our principal sonographer Ms Vasanthy Gurumurthy for her contribution.

\section{REFERENCES}

1. Hsu LY, Chia PY, Lim JF. The Novel Coronavirus (SARS-CoV-2) Epidemic. Ann Acad Med Singapore 2020;49:105-7.

2. Lai J, Ma S, Wang Y, Cai Z, Hu J, Wei N, et al. Factors Associated With Mental Health Outcomes Among Health Care Workers Exposed to Coronavirus Disease 2019. JAMA Netw Open 2020;3:e203976.

3. Goh KT, Cutter J, Heng BH, Ma S, Koh BK, Kwok C, et al. Epidemiology and control of SARS in Singapore. Ann Acad Med Singapore 2006;35:301-16.

4. Committee on Practice Bulletins-Obstetrics and the American Institute of Ultrasound in Medicine. Practice Bulletin No. 175: Ultrasound in Pregnancy. Obstet Gynecol 2016;128:e241-e256.

5. Abu-Rustum RS, Akolekar R, Sotiriadis A, Salomon LJ, Da Silva Costa F, Wu Q, et al. ISUOG Consensus Statement on organization of routine and specialist obstetric ultrasound services in the context of COVID-19. Ultrasound Obstet Gynecol 2020;55(6):863-870.

6. American Institute of Ultrasound in Medicine. Guidelines for Cleaning and Preparing External- and Internal-Use Ultrasound Transducers Between Patients, Safe Handling, and Use of Ultrasound Coupling Gel2018 [Available from: https:/www.aium.org/accreditation/ Guidelines_Cleaning_Preparing.pdf.)

7. Poon LC, Yang H, Lee JCS, Copel JA, Leung TY, Zhang Y, et al. ISUOG Interim Guidance on 2019 novel coronavirus infection during pregnancy and puerperium: information for healthcare professionals. Ultrasound Obstet Gynecol 2020;55(5):700-708.

8. Chen M, Leo YS, Ang B, Heng BH, Choo P. The outbreak of SARS at Tan Tock Seng Hospital--relating epidemiology to control. Ann Acad Med Singap 2006;35:317-25. 